\title{
PVP2005-71291
}

\section{ADVANCED DESIGN OF PACKING AND CYLINDERS FOR HYPER-COMPRESSORS FOR LDPE PRODUCTION}

\author{
Enzo Giacomelli \\ GE Energy, Nuovo Pignone \\ Global Services \\ Florence, Italy
}

\author{
Franco Graziani \\ GE Energy, Nuovo Pignone \\ Engineering \\ Florence, Italy
}

\author{
Simone Pratesi \\ GE Energy, Nuovo Pignone \\ Engineering \\ Florence, Italy
}

Giovanni Zonfrillo

University of Florence

Dept. of Mechanical and Industrial Technology

Florence, Italy

\author{
Iacopo Giovannetti \\ University of Florence \\ Dept. of Mechanical and Industrial Technology \\ Florence, Italy
}

\begin{abstract}
The optimization of packing cup geometry is important for improving the reliability of hyper-compressors. The complex arrangement and the transmission of loads through the various components requires the use of FEA to properly determine the stress level.

The need to reduce the sensitivity to external factors requires an investigation with more advanced simulations.

The entire cylinder and associated components such as head flanges, cylinder chambers and tie rods, were modeled. This provided the characteristics of the interfaces and the boundary conditions necessary to simulate the packing cups. Considering the thickness, ring housing profile, lube-oil holes, mating surfaces, interference-fit and diameter make it possible to obtain the stresses at the most critical points.

This new simulation approach shows the effect of the parameters that influence the stress level at critical points, thus optimizing the packing cup profile and its relevant features.
\end{abstract}

